\title{
Modified Miccoli's thyroid surgery for thyroid diseases
}

\author{
HUI YU ${ }^{1}$, XIN GE $^{1}$, WEIKANG PAN ${ }^{1}$, HUAIJIE WANG ${ }^{1}$, QIANG HUANG ${ }^{1}$, \\ YU DONG ${ }^{2}$, YA GAO ${ }^{1}$ and JIANJUN YU ${ }^{2}$ \\ ${ }^{1}$ Department of Pediatric Surgery, The Second Affiliated Hospital, School of Medicine, Xi'an Jiaotong University, \\ Xi'an, Shaanxi $710004 ;^{2}$ Department of Minimally Invasive Surgery, The People's Hospital \\ of Ningxia Hui Autonomous Region, Yinchuan, Ningxia 750001, P.R. China
}

Received March 13, 2015; Accepted June 4, 2015

DOI: $10.3892 / \mathrm{mco} .2015 .597$

\begin{abstract}
Minimally invasive video-assisted thyroidectomy (MIVAT), originally described by Miccoli, is considered to be the most widely practiced and easily reproducible procedure for selected patients with benign and/or malignant thyroid nodules. Modified techniques based on MIVAT, namely modified Miccoli's thyroid surgery (MMTS), were developed based on MIVAT. This study aimed to evaluate the preliminary results of MMTS compared with those of MIVAT. The enrolling criteria included a benign nodule $<3.5 \mathrm{~cm}$ in diameter, a malignant tumor $<2 \mathrm{~cm}$, no previous neck surgery and no evidence of any suspected lymph node metastasis or local invasion. Unilateral lobectomy was considered for benign lesions and the additional dissection of central compartment (level VI) lymph nodes was applied for malignant disease. The modified techniques included carefully selecting the operative incision, expanding the operative space, embedding a drainage tube in situ and delicately suturing every layer inwards and crosswise, as well as measuring cervical motion. In addition to the comparison of surgical outcomes between MMTS and MIVAT, other surgical parameters, including operative time, blood loss, postoperative drainage, cosmetic satisfaction, peak angle of cervical rotation, length of hospitalization and complications, were retrospectively analyzed. A consecutive series of 70 patients, including 54 cases of benign and 16 cases of malignant disease, initially underwent MIVAT between April, 2008 and May, 2012, while 127 patients, including 98 benign and 29 malignant cases, subsequently underwent MMTS between September, 2011 and October, 2014. Patients who received MMTS exhibited significantly less blood loss
\end{abstract}

Correspondence to: Dr Ya Gao, Department of Pediatric Surgery, The Second Affiliated Hospital, School of Medical, Xi'an Jiaotong University, 157 Xiwulu, Xi'an, Shaanxi 710004, P.R. China

E-mail:ygao@mail.xjtu.edu.cn

Dr Jianjun Yu, Department of Minimally Invasive Surgery, The People's Hospital of Ningxia Hui Autonomous Region, 301 Zhengyuan North Street, Yinchuan, Ningxia 750001, P.R. China E-mail: yujjster@gmail.com

Key words: modified surgical techniques, thyroidectomy, modified Miccoli's thyroid surgery, cosmetic result, advantages
(20.3 \pm 11.3 vs. $32.3 \pm 12.6 \mathrm{ml}, \mathrm{P}<0.01)$, lower volume of postoperative drainage $(42.77 \pm 15.2$ vs. $50.48 \pm 23.2 \mathrm{ml}, \mathrm{P}<0.01)$ and higher cosmetic satisfaction $(94.6 \pm 3.5$ vs. $88.9 \pm 2.7 \%, \mathrm{P}<0.01)$, but a longer operative time $(102 \pm 36$ vs. $50.48 \pm 23.2 \mathrm{~min}, \mathrm{P}<0.01)$ when compared with MIVAT. In addition, a better peak angle of cervical rotation $\left(38.6 \pm 4.1^{\circ}\right.$ vs. $\left.35.3 \pm 3.8^{\circ}, \mathrm{P}=0.25\right)$ and shorter length of hospitalization ( $4.25 \pm 1.08$ vs. $4.51 \pm 1.30$ days, $\mathrm{P}=0.52)$ was observed in the MMTS group, although the differences with the MIVAT group were not statistically significant. No complications were observed, apart from 2 cases of recurrent laryngeal nerve palsy and 1 case of transient hypocalcemia at the beginning of MIVAT. In conclusion, both MMST and MIVAT are safe and feasible methods of thyroidectomy; however, MMST is associated with less trauma and higher cosmetic satisfaction compared with MIVAT. Therefore, MMST may be used as a standard operative method and prospectively applicable for thyroidectomy, even for early-stage malignancies.

\section{Introduction}

Minimally invasive techniques for thyroidectomy have applied various endoscopic approaches, such as through two or more holes (1), the axillo-bilateral-breast approach (2) and the anterior chest wall approach (3) after the first endoscopic parathyroidectomy in 1996. MIVAT, originally described by Miccoli, has gradually been accepted due to its advantages, such as better cosmetic results compared with traditional procedures and better postoperative outcome, as well as lower medical cost (4-6). The range of applications of MIVAT has expanded rapidly from benign disease to malignancy.

However, the MIVAT procedure has been associated with certain disadvantages, such as limited operative space, use of a thick rubber drainage tube and keloid formation. To address these issues, effective surgical techniques have been investigated; for example, a better operative space may be provided by using a modified retractor. Moreover, placement of a smaller drainage tube of moderate hardness has replaced thick rubber tubes for routine drainage; in addition to carefully selecting the operative incision and suturing every layer inwards and crosswise, sticky dressing was applied for tension-free repair.

The aim of this study was to retrospectively compare the results between patients who underwent MMST and those who underwent MIVAT, in order to establish the safety, feasibility and aesthetic superiority of MMST. 
A

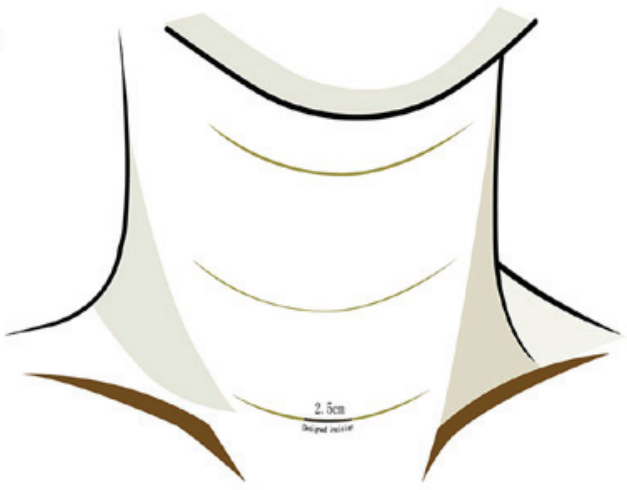

C

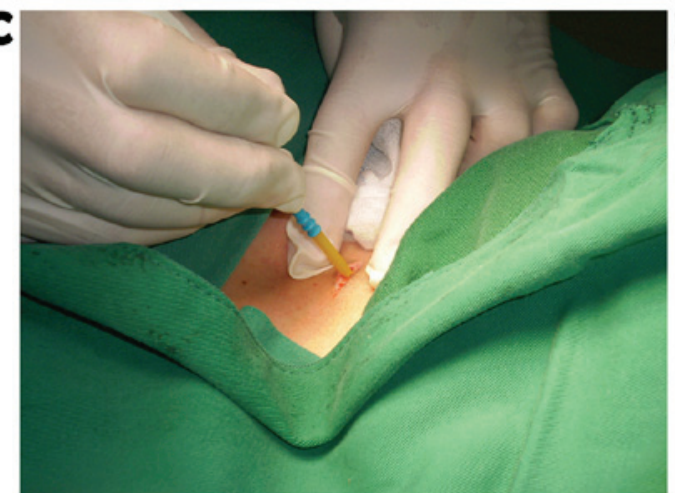

$\mathbf{E}$

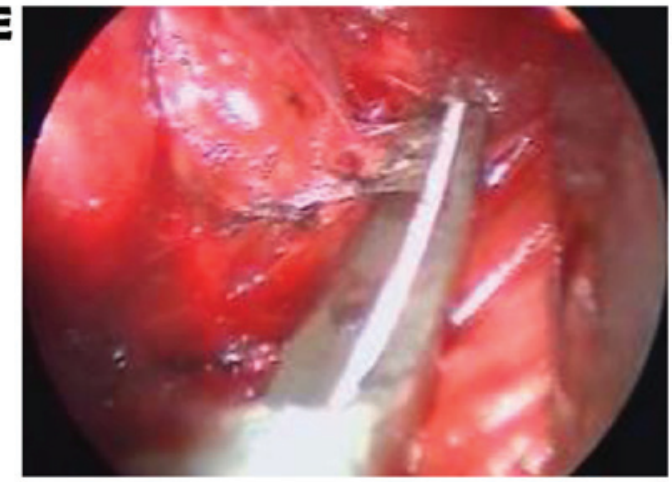

B
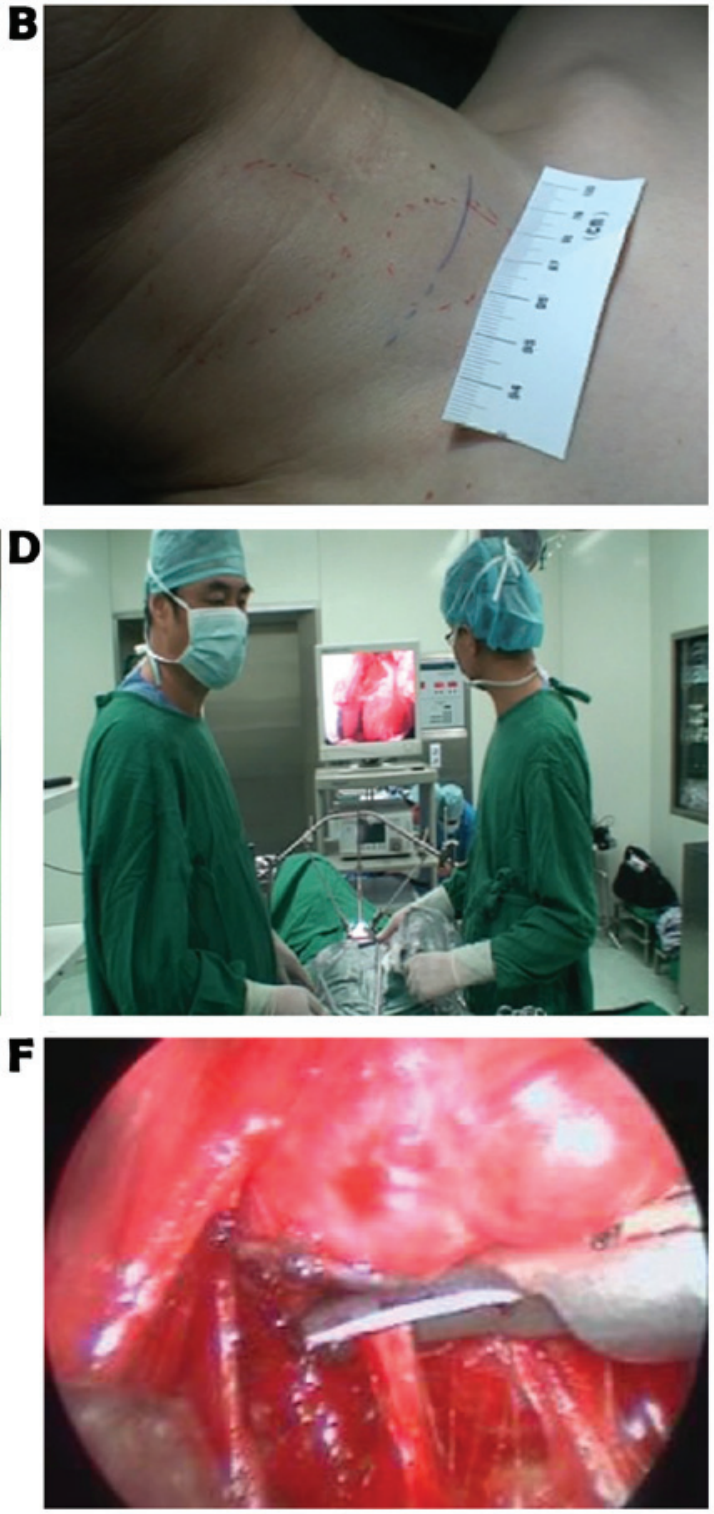

Figure 1. (A) Schematic illustration of the modified Miccoli's thyroid surgery (MMTS) incision in the upright position. (B) The incision is designed to coincide symmetrically with the last prominent line of Langer. (C) A needle-like electrotome with a rubber tube is used for the MMTS incision and hemostasis. (D) Intraoperative view of the gasless endoscopic setup. (E and F) The procedure was performed under endoscopic vision and the harmonic scalpel facilitated resection and extraction of the lobe.

\section{Patients and methods}

Patients and criteria. A total of 70 consecutive patients who underwent MIVAT between April, 2008 and May, 2012 and 127 patients who underwent MMTS between September, 2011 and October, 2014 were enrolled in this study. The eligibility and exclusion criteria were as previously described (7).

Preoperative preparations. All the patients underwent thyroid ultrasound and functional examinations in order to exclude hyperthyroidism, hypothyroidism and hyperactive adenoma. A methoxyisobutylisonitrile scan and enhanced computed tomography were also performed to exclude any suspected lymph node metastasis or local invasion. In all the cases, an intraoperative frozen section examination was performed and the paraffin-embedded sections were routinely examined postoperatively.

\section{Modified surgical techniques}

Selecting the operative incision. The incision was routinely designed in detail to coincide with last prominent line of Langer (Fig. 1A and B), $\sim 3.0 \mathrm{~cm}$ or two fingers above the sternal notch. The primary requirement of the designed incision was to move symmetrically along the last prominent line of Langer (Fig. 1C).

Expanding the operative space. A gasless lifting system with modified retractors was installed, suspended and fixed in an orderly manner (Fig. 1D). Sufficient working space was created through a $30^{\circ}(5 \mathrm{~mm})$ endoscope. The procedure was performed under endoscopic vision, with an amplified video (Fig. 1E and F) to help discriminate between different anatomical structures.

Suturing each layer inwards and crosswise. The Hunter line, fascia, platysma and subcutaneous tissue were tightly sutured inwards and crosswise (Fig. 2A) following conventional 

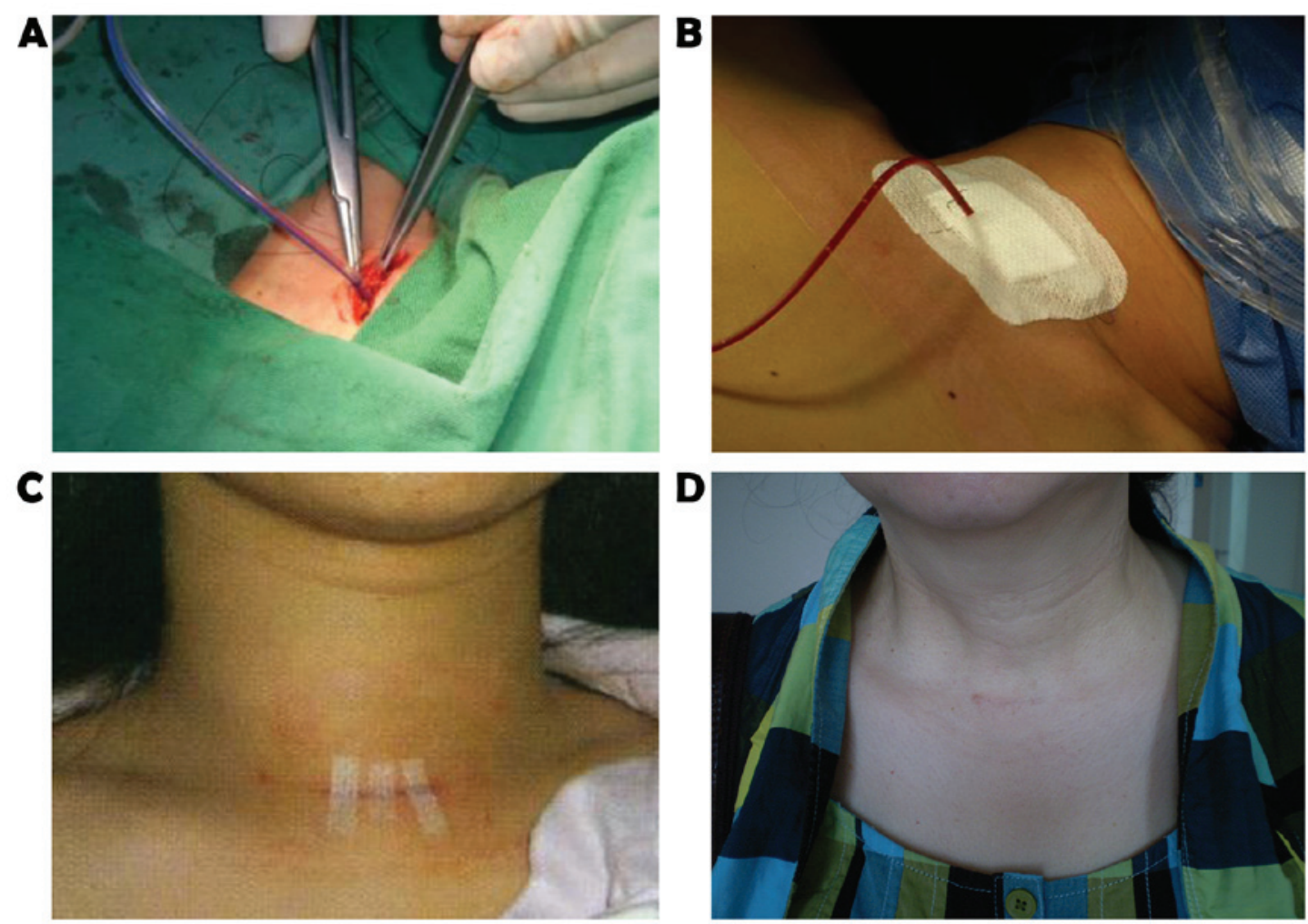

Figure 2. (A and B) A drainage tube was carefully placed in situ and each layer was sutured inwards and crosswise. (C) Cosmetic result of modified Miccoli's thyroid surgery on the 3 rd postoperative day. (D) The scar is barely visible 1 month postoperatively.

check-up. The skin was closed through subcuticular suturing and sticky dressing was applied to achieve tension-free repair (Fig. 2B). The joined points of the adjacent layers were not in the same perpendicular line, but rather crosswise-positioned.

Placement of the drainage tube in situ. A small tube of moderate hardness, connected to a compressible oval bulb, partly used to maintain an air-free state for effective drainage, was carefully embedded in situ. The steps were similar to those during conventional procedures.

Measuring cervical motion. The method for measuring the precise cervical range of motion was previously reported by our team (7).

Statistical analysis. All the data are presented as means \pm standard deviation. Group data from the MIVAT and MMTS groups were analyzed. The non-parametric Mann-Whitney test was used to compare the means of the parameters. A value of $\mathrm{P}<0.01$ was considered to indicate a statistically significant difference.

\section{Results}

Patients. A consecutive series of 70 patients, including 54 benign and 16 malignant cases, initially underwent MIVAT between April, 2008 and May, 2012, whereas 127 patients, including 98 benign and 29 malignant cases, subsequently underwent MMTS between September, 2011 and October, 2014. The preoperative characteristics were similar between the MMTS and MIVAT groups (Table I).

Surgical results. General anesthesia was applied to all the patients and no cases were converted to conventional
Table I. Clinical characteristics of all the patients undergoing thyroid surgery.

\begin{tabular}{lcc}
\hline & MIVAT & MMST \\
Characteristics & $(\mathrm{n}=70)$ & $(\mathrm{n}=127)$ \\
\hline
\end{tabular}

\begin{tabular}{lcc}
\hline Gender & & \\
F\% (F/M) & $77.14(54 / 16)$ & $77.95(99 / 28)$ \\
Age (years) & & \\
Mean \pm SD & $45 \pm 9$ & $46 \pm 10$ \\
Range & $22-60$ & $22-65$
\end{tabular}

Max tumor

diameter $(\mathrm{cm})$

$\begin{array}{lll}\text { Mean } \pm \mathrm{SD} & 1.8 \pm 0.9 & 1.8 \pm 0.8 \\ \text { Range } & 0.2-3.5 & 0.2-3.5\end{array}$

Location of tumors

$\mathrm{L} \%(\mathrm{R} / \mathrm{L})$

$54.29(38 / 32)$

$53.54(68 / 59)$

MIVAT, minimally invasive video-assisted thyroidectomy; MMTS, modified Miccoli's thyroid surgery; F, female; M, male; SD, standard deviation; L, left lobe; R, right lobe.

thyroidectomy. All the MMTS and MIVAT procedures were performed by the same surgeon. The surgical parameters, including operative time, blood loss, postoperative drainage, peak angle of cervical rotation, length of hospitalization and cosmetic satisfaction, are listed in Table II. Two cases of recurrent laryngeal nerve palsy and 1 case of transient 
Table II. Intraoperative and follow-up data.

\begin{tabular}{lccc}
\hline Surgical parameters & MIVAT $(\mathrm{n}=70)$ & MMST (n=127) & P-value \\
\hline Operative time (min) & $82 \pm 29$ & $102 \pm 36$ & $<0.01$ \\
Blood loss (ml) & $32.3 \pm 12.6$ & $20.3 \pm 11.3$ & $<0.01$ \\
Postoperative drainage (ml) & $50.48 \pm 23.2$ & $42.77 \pm 15.2$ & $<0.01$ \\
Peak angle of cervical rotation $\left(^{\circ}\right)$ & $35.3 \pm 3.8^{\circ}$ & $38.6 \pm 4.1^{\circ}$ & 0.25 \\
Length of hospitalization (days) & $4.51 \pm 1.30$ & $4.25 \pm 1.08$ & 0.52 \\
Cosmetic satisfaction $(\%)$ & $88.9 \pm 2.7$ & $94.6 \pm 3.5$ & $<0.01$ \\
\hline
\end{tabular}

The data are presented as means \pm standard deviation. MMTS, modified Miccoli's thyroid surgery; MIVAT, minimally invasive video-assisted thyroidectomy.

hypocalcemia at the beginning of MIVAT recovered following symptomatic treatment. A 12-month follow-up was required, including thyroid ultrasonography and function. The serum thyroglobulin level was measured in patients with malignant lesions. The cosmetic results of MMTS were visible on the 3rd day (Fig. 2C) and the 1st month (Fig. 2D) postoperatively.

\section{Discussion}

Although MIVAT has several advantages, including less postoperative pain, shorter hospital stay, more attractive cosmetic results and reduced distress (8) through transforming growth factor- $\beta$ serum levels, as well as no additional risks of rupturing the capsule with subsequent cancer cell dissemination (9) and and other thyroidectomy complications, it also has certain disadvantages, such as narrow operative space, use of a thick rubber drainage tube and keloid formation. Based on MIVAT, MMST uses modified surgical techniques to achieve better results and cosmetic satisfaction.

The issue of the thyroidectomy incision was investigated several decades prior. Desault first initiated an anterior median longitudinal incision; next, a similarly lateral incision but parallel to the inner border of the sternocleidomastoid muscle was planned by Billroth; his junior, Kocher, established an 8-10 cm collar incision that became popular in the 20th century (10). Recently, Miccoli introduced the MIVAT procedure (11), which revolutionized minimally invasive thyroidectomy. A $1.5-\mathrm{cm}$ horizontal incision placed $2 \mathrm{~cm}$ above the sternal notch is associated with less trauma, compared to other approaches. However, the standards of the incision have not been clearly determined, except for a study reporting that, if placed $<2 \mathrm{~cm}$ or one fingerbreadth away from the sternal notch, particularly on the manubrium, the incision may result in a hypertrophic scar or keloid (12). In addition, a precise measurable approach was introduced to determine optimal incision placement between the cricoid cartilage and the sternal notch (13). By contrast, another previous study indicated that the incision migrates in different positions (14). Of note, migration of the incision of $\sim 9 \mathrm{~mm}$ downwards was observed during postoperative follow-up when changing the body position from upright to supine in a preliminary study (15). Furthermore, cosmetic principles suggest that the remaining incision may result in a hypertrophic scar or keloid when deviating away from the Langer's line and recommend using pre-existing neck creases to better hide the incisions. Therefore, the incision in our department was designed to coincide with the last prominent Langer's line, between the cricoid cartilage and the sternal notch, and was individualized as required.

In practice, cervical motion is considered to be negatively correlated with postoperative pain, and may be a useful parameter to monitor functional recovery; however, this is not a simple and rapid way to evaluate drainage, incision length and duration of hospitalization in clinical practice and only a limited number of studies consider this parameter. The quantifiable angle from the submental area to the maximal degree bilaterally was noted after our patients were encouraged to move their heads, which may be used to measure injuries directly.

Despite the possibility of drainage tube-related damage to the laryngeal nerve and tube blocking by clots, drainage is crucial for preventing hematoma formation from the slow extravasation of blood and lymph fluid in a closed and narrow space. Placing a smaller drainage tube in the thyroid bed is now routinely practiced. Moreover, the air-free state of the smaller tube makes drainage more effective from each layer.

Finally, as an independent indicator reported by Sabuncuoglu et al (16), scar tissue formation of $<3 \mathrm{~cm}$ is important for cosmetic satisfaction. Therefore, it is crucial for clinicians to study the characteristics of keloid formation.

In conclusion, MMTS is feasible and safe for the treatment of benign and/or malignant thyroid nodules, similar to MIVAT. Furthermore, MMST is associated with less trauma and higher cosmetic satisfaction compared with MIVAT. However, further investigation is required to confirm the long-term outcome and more attention should be focused on keloid formation.

\section{References}

1. Pai SI and Tufano RP: Central compartment neck dissection for thyroid cancer. Technical considerations. ORL J Otorhinolaryngol Relat Spec 70: 292-297, 2008.

2. Bärlehner E and Benhidjeb T: Cervical scarless endoscopic thyroidectomy: Axillo-bilateral-breast approach (ABBA). Surg Endosc 22: 154-157, 2008.

3. Nakano S, Kijima Y, Owaki T, Shirao K, Baba M and Aikou T: Anterior chest wall approach for video-assisted thyroidectomy using a modifed neck skin lifting method. Biomed Pharmacother 56 (Suppl 1): 96S-99S, 2002. 
4. Timon C and Miller IS: Minimally invasive video-assisted thyroidectomy: Indications and technique. Laryngoscope 116: 1046-1049, 2006.

5. Lombardi CP, Raffaelli M, Princi P, De Crea C and Bellantone R: Video-assisted thyroidectomy: Report on the experience of a single center in more than four hundred cases. World J Surg 30: 794-801, 2006.

6. Miccoli B, Berti P, Bendinelli C, Conte M, Fasolini F and Martino E: Minimally invasive video-assisted surgery of the thyroid: A preliminary report. Lange becks Arch Surg 385: 261-264, 2000

7. Yu JJ, Bao SL, Yu SL, Zhang DQ, Loo WT, Chow LW, Su L, Cui Z, Chen K, Ma LQ, et al: Minimally invasive video-assisted thyroidectomy for the early-stage differential thyroid carcinoma. J Transl Med 10 (Suppl 1): S13, 2012.

8. Elaraj DM and Clark OH: Changing management in patients with papillary thyroid cancer. Curr Treat Options Oncol 8: 305-313, 2007.

9. Miccoli P, Berti P, Raffaelli M, Materazzi G, Baldacci S and Rossi G: Comparison between minimally invasive video-assisted thyroidectomy and conventional thyroidectomy: A prospective randomized study. Surgery 130: 1039-1043, 2001.
10. Thompson NW, Olsenn WR and Hoffman GL: The continuing development of the technique of thyroidectomy. Surgery 73 913-927, 1973.

11. Pinchot S, Chen H and Sippel R: Incisions and exposure of the neck for thyroidectomy and parathyroidectomy. Operat Tech Gen Surg 10: 63-76, 2008.

12. Scott-Conner CE and Dawson DL (eds): Operative anatomy. (1st edition). JB Lippincott Company, Philadelphia, PA, p388, 1993.

13. Xiao GZ and Gao L: A simple method for determining an optimal incision for minimally invasive video-assisted thyroidectomy. Surg Endosc 22: 2100-2101, 2008.

14. Jancewicz S, Sidhu S, Jalaludin B and Campbell P: Optimal position for a cervical collar incision: A prospective study. ANZ J Surg 72: 15-17, 2002.

15. Yu H, Yang YZ, Zhang DQ, Cui Z and Yu JJ: Analysis of cervical dermatoglyph and related factors. Chin Arch Gen Surg 4: 571-572, 2010.

16. Sabuncuoglu MZ, Sabuncuoglu A, Sozen I, Benzin MF, Cakir T and Cetin R: Minimally invasive surgery using mini anterior incision for thyroid diseases: A prospective cohort study. Int J Clin Exp Med 7: 3404-3409, 2014. 